\title{
Research on Model-Driven Simulation Approach for Healthcare Information System
}

\author{
L. L. Song, X. Q. Guo \\ Wuhan General Hospital of Guangzhou Command \\ Wuhan Hubei, China
}

\author{
C. Wang \\ 75709th troops of Chinese People’s Liberation Army \\ Wuhan Hubei, China
}

\begin{abstract}
The project has studied the model-driven simulation approach for healthcare information integration system design analysis and optimization, which includes simulation model specification design, transformation of reference information model (RIM) to simulation model, and integrated simulation supporting framework. Simulation model specification is studied and formulated based on simulation model portability 2.0 (SMP2) to extend healthcare information system development methodology (HDF) and enhance the simulation modeling ability; Transformation of RIM to SMP2 platform-independent simulation model, modeling tools and codes (or code framework) automatically generation is implemented based on meta-modeling technique to realize the simulation model automatic or semiautomatic generation. Finally, the executable simulation approach for healthcare information system is realized.
\end{abstract}

Keywords-healthcare information system; simulation framework; model-driven; HDF; SMP2

\section{INTRODUCTION}

With the rapid development of medical technology and medical equipments, medical and health management is more complex and difficult, and the health information system has become an important way to improve medical quality and efficiency and realize the scientific management of medical affairs. Especially in recent years, with China's medical and healthcare system reform deepen and hospitals and medical institutions being developed in the direction to the enterprise, the information system scale has experienced four phases: single computer system with single user, department level application, hospital level application, and regional medical collaboration, the connotation of HIS is evolved from original hospital information systems to healthcare information systems, which has advanced further requirements to system interoperability. In general, there still exist the following several problem in our health information construction [1-2].

(1) Due to the lack of uniform standards and specification, the medical information system from the bottom to the data model and implementation technology, business processes and data dictionary are heterogeneous.

(2) The development of China's medical and health information is unbalance, and the information construction of many basic medical units is not mature enough, no matter from the depth or breadth. Information systems is customized according to their own needs, and the semantic information, such as business process, data concepts abstract, is hidden and fixed in the program, which is unfavorable to system scalability and interoperability.

(3) Most existing medical information system are focused on implementation of information digitalization and business process automation to improve medical quality and efficiency, but there is still a big gap to realize scientific and standardized management of medical affairs, such as medical science, business restructuring, clinical pathway management optimization, resource optimization configuration.

(4) Health information system integration especially the regional medical collaboration often involves multiple medical institutions, and there is still lack of scientific and systematic methods and effective tool to guide and support the design and optimization of medical business or management process.

\section{RELATED WORKS}

In recent years, many new technologies like distributed component technology, workflow technology and Web Service have been widely applied in the field of enterprise integration, which also has been widespread concerned in the medical information field [3-4]. To develop the medical information standards are paid attention by the relevant departments, national health ministry has published a number of health information standards, such as health information element directory, and follow the international or national medical data standard such as HL7 (Health Level Seven) V2, DICOM has become the basic requirements of the medical information system development. Especially in 2005, the HL7 organization has launched XML based versions of V3 [5-6], which has absorbed the new technology and new methodology of the information and Computer Science (such as service oriented architecture of SOA, semantic Web, model driven architecture MDA), and put forward the development methodology of health information system - HL7 development framework HDF. The framework is to describe health information system, so as to provide a clear blueprint about system -- what kind of conditions is needed to interoperate with it for external users! Now HL7 V3 and HDF have been widely recognized in the industry, and are being researched in China. HL7 V3 and HDF are mainly for health information system design and development, so as to support user consistent understanding of system and correct use, which can 
be very good to solve present above (1) and (2) problems, but insufficient for (3) and (4) problems.

In view of (3) and (4) problems, researchers [7-8] has analyzed medical business process from the point of view of model test to support medical guide design. This kind of method generally requires higher normal description techniques, which are difficult to describe complex process, and do not pay attention to management optimization problems (such as business restructuring, resource allocation problem). Some other researchers [9-10] have made quantitative analysis for related department of hospital to provide the basis for the allocation of resources and business process optimization utilizing modeling and simulation. The clinical path modeling and simulation method based on Petri net is provided to optimize the resource allocation in execution of clinical path [11-12] A discrete event simulation method was put forward to analyzed surgical operation department, which can used to evaluate and optimize clinical pathway [1315]. The main problem of these methods is that, special simulation skills and tools are needed, which is unfavorable for users.

Therefore we propose a model driven approach for health information system simulation, and the key technologies are discussed. The core idea of model driven is that the model representation is abstracted from specific environment and platform to build a platform independent model, and through the model transformation tools and code generation tools to generate platform dependent code. "Model driven" contains two meanings: one is to extend HDF by adding corresponding simulation elements information to establish the simulation model specification; two is the automatic or semi-automatic mapping of extended HDF based design models on simulation models. The goal is to realize the automation of health information system simulation, so designers only need to pay attention to the realization of the business logic, do need not to master the relevant simulation skills to complete the design plan demonstration, system optimization analysis and system test application demand of the health information.

\section{MODEL DRIVEN SIMULATION APPROACH FOR HEALTHCARE INFORMATION SYSTEM}

\section{A. Methodologies}

The essence of "Model Driven" is to realize the automation of health information system simulation by making use of method and technology of model driven architecture (MDA). MDA[16] is an advanced software engineering method proposed by object management group OMG in 2001, also known as model driven engineering, model driven development (Design), which provides related technical standards, such as element modeling specification MOF (meta object facility), model storage and interchange specification XMI (XML metadata Interchange), modeling language UML model transformation language, QVT (query / view / transform) etc.. MDA has advocated at the model level (rather than code level) to solve integration between different software tools, and the basic idea is not to construct the unified modeling language and it's supporting technology standards, but to achieve automatic generation of domain oriented modeling language and modeling environment based on metamodeling technologies. Therefore, MDA method and related techniques can provide comprehensive support for the realization of model transformation, modeling tool, and automatic generation of code framework. The process reference model of healthcare information system simulation analysis based on MDA is shown as figure 1.

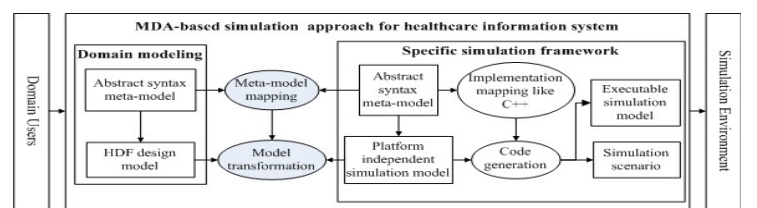

FIGURE I. PROCESS REFERENCE MODEL OF MDA-BASED SIMULATION APPROACH FOR HEALTHCARE INFORMATION SYSTEM.

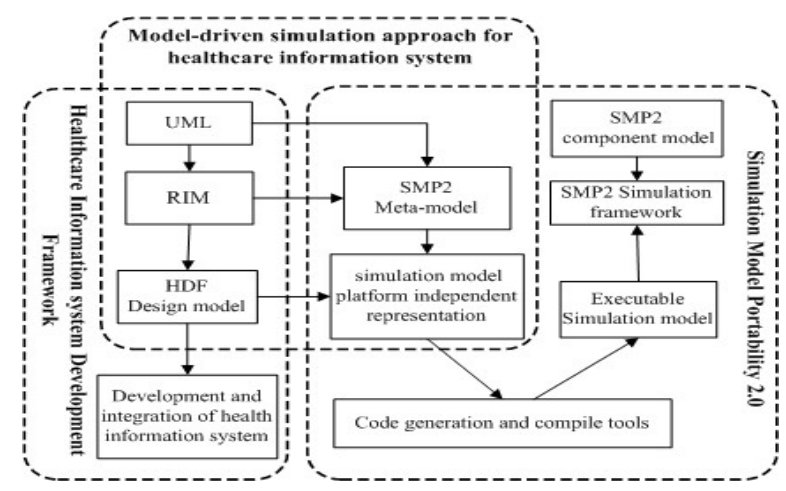

FIGURE II. RELATIONSHIP AMONG MODEL DRIVEN SIMULATION APPROACH FOR HEALTHCARE INFORMATION SYSTEM, HDF AND SMP2.

Comprehensive comparison of different simulation methods and simulation framework, Simulation model portability standard version 2 (SMP2) is adopted to extend the HDF. SMP2 [17] is the integration of software engineering and M\&S (modeling and simulation), and is the application of MDA in the field of M\&S. SMP2 mainly includes three parts: SMP2 meta-model, SMP2 component model and SMP2 C++ mapping. SMP2 meta-model is also called simulation model definition language, which can be used to design platform independent model, create model instance and its interaction, and the scheduling scheme for instances; SMP2 component model has defined platform independent interface between simulation model and simulation environment, including the model component interface, simulation service component interfaces, simulator component interface, so that the SMP2 simulation model and the SMP2 simulation environment can be independently developed and automatic integration; SMP2 $\mathrm{C}++$ mapping has provided the $\mathrm{C}++$ code generation template for SMP2 meta-model and SMP2 component model, which can support the development of executable simulation model. Fig.2 has clearly identified the relationship among model driven simulation methodology for healthcare information system, the HDF healthcare information systems development 
methodology and the SMP2 simulation development methodology.

\section{B. Key Technologies}

1) Simulation model specification for healthcare information system: HL7 HDF is the information system development methodology for healthcare domain science; fully absorb the MDA methodology and technologies in the field of software engineering. HDF has emphasized on the standard description based on XML language, the usage of UML graphical modeling language to design the health information system, and the greatest contribution is the establishment of an information model in medical field-RIM (Reference Information Model). RIM is a mature static model about medical application information, which has defined comprehensive description model for medical information and standardized description for medical behaviors and was admitted to the ISO standard in 2006. But RIM is still designoriented, and can only describe the structure, behavior and usage information about systems. HDF has used UML as modeling language, which lacks the description of basic simulation elements, such as time management, event scheduling, synchronization control, and instance modeling capabilities are relatively weak.

But SMP2 is a specification with lower abstract level, and its modeling concepts (such as class, component, interface and so on) are very technical, mixing with the complexity of MDA, which is difficult for domain modeling personnel to understand and accept. So more efficient modeling views oriented different domains are needed to be developed based on SMP2, namely further integrated several typical health requirements with existing HDF fruits, the simulation model specification for healthcare is developed based on SMP2 to extend $\mathrm{HDF}(\mathrm{HDF}+)$ and enhance the simulation modeling ability of HDF.

2) Transformation of HDF design model to SMP2 simulation model: Transformation and mapping of design model to simulation model is to realize the automatic (semiautomatic) generation of simulation model, which mainly includes two aspects. One is the mapping of the platform independent HDF design model to specific simulation model specification (i.e. SMP2); the other is the platform independent model to the platform dependent model(such as programming language, operating system, simulation framework) mapping. SMP2 meta-model is defined by UML for simulation domain, and the HDF design model is also established based on UML. The core of HDF is reference information model RIM, which is a specific domain model for the medical field. SMP2 is a low-level specification, not for any specific application domain. These two model framework (RIM and SMP2) correspond to different conceptual systems and need to be conversed, and the conversion will be carried out according to the following steps:

(1) Design the mapping rules between RIM and SMP2 simulation model based on the QVT;

(2) Transform based on QVT engine;

(3) Generate the executable simulation model based on SMP2 code generator.

3) Integrated simulation framework for healthcare information system: The main function of integrated simulation framework for healthcare information system is the integration of above research key problems under the guidance of MDA, and provides the modeling and simulation tools for health information systems analysis tools. This framework mainly relates to domain modeling user and information system designer: domain modeling user is responsible for the establishment of medical business model, and information system designer is responsible for building information system design model and realizing of the business logic. With the development of medical information system, the medical institutions have applied various medical information systems according to their own characteristics, and they may need different simulation analysis for the same medical information system according to different purposes (medical efficiency, cost etc.). So, the unified information system modeling mechanism and flexible simulation mechanism are needed, which focus on following contents:

(1) The simulation model scheduling mechanism, assembly and scheduling method for several simulation model of typical medical information system are researched;

(2) Graphical modeling tool, including simulation modeling tool, assembly tool and scheduling model design tool;

(3) Import of the design model based on UML;

(4) Simulation model scheduling engine;

(5) Model and simulation resources (such as simulation lock, queue) management tool.

\section{SERVICE-ORIENTED INTEGRATED SIMULATION} PLATFORM FOR HEALTHCARE INFORMATION SYSTEM

\section{A. Architecture}

The main function of the integrated simulation platform for healthcare information systems is the integration of tools. Considering the requirements of regional collaboration, the service oriented integration simulation platform architecture is advanced, which is shown as figure 3 . The platform can support distributed collaborative development and simulation test by using Web Service technologies. Domain modeling user can do model design, simulation development and analysis in local, and can also do this through the Web Service communication interface to complete integrated design. The simulation model and the integrated application system can invoke the remote service with Web Service interface. 


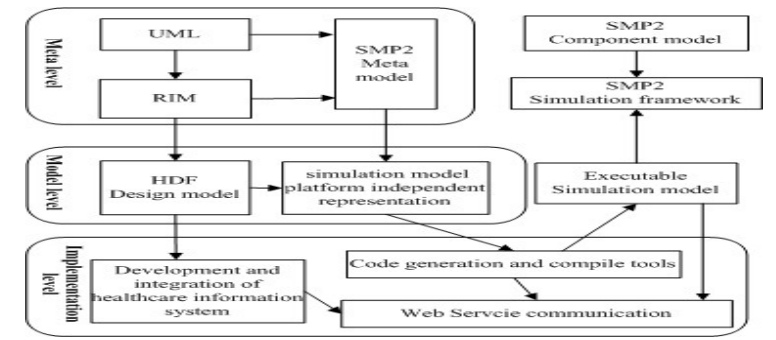

FIGURE III. RELATIONSHIP AMONG MODEL DRIVEN SIMULATION APPROACH FOR HEALTHCARE INFORMATION SYSTEM, HDF AND THE SMP2.

\section{B. Implementation}

1) Simulation model scheduling engine: The advantage of SMP2 specification lies in three separations: separation of simulator with simulation service, platform independent model PIM with platform specific model PSM, and the design component with runtime component, which has made flexible and variable simulation possible. The simulation model scheduling engine is the implementation of simulation mechanism, and is the core component of simulation execution, which is responsible for driving simulation on base of model framework, assembly design and scheduling design. The simulation will be carried out in accordance with the following procedures:

(1) Interpret of assembly model and scheduling model following XML format;

(2) According to model framework and assembly model, specific models are selected and initialized in the model library, and the model interactive relationship is established;

(3) According to the scheduling model the model scheduling events are initialized and placed in the event queue to be processed;

(4)Pop the incident with the smallest time stamp from event queue and process;

(5) Advance simulation time;

(6) Return to step 4 until the pending queue is empty or the simulation time has reached a maximum value.

2) Generation of graphical modeling tool based on Eclipse GMF: It is costly to develop specific modeling tool for modeling language, but meta-modeling techniques of MDA is one of the way to solve this problem. Through meta-modeling, the domain modeling language can be customized according to application fields, and then the modeling tool can be automatically generated for the modeling language. The MDA MOF has defined a meta-model description specification, and the Eclipse Graphical Modeling Framework GMF is one implementation of meta-modeling technology, which has provided a powerful framework for model-based development of graphical editor. Visual simulation modeling tool can be designed based on GMF for field user.
3) Generation of code based on freemarker: FreeMarker is a template mapping technology, which is also a template based code generation tool. But it is not oriented end users, but a tool provided for developers. The basic idea of FreeMarker is through a data model and template to output code. Data model is a tree data structure in memory, while the template is some basic content written with fixed format in advance, in which the blank position or the content marked with placeholder is filled with specific data model (data model is defined by the user). The placeholders has defined according to the node name in data model, and the node value would be used replace the placeholders when generating codes. FreeMarker has realized the separation of presentation layer and business logic, which makes a clearer division of labor and greatly improves the development efficiency.

\section{CONCLUSIONS}

This paper has fully absorbed and learnt from the achievements of healthcare information and simulation domain, further has strengthened the research of simulation engineering approach and key technologies for the design and analysis of healthcare information system from point of system engineering. The simulation model specification for medical business, model mapping specification, simulation model scheduling mechanism, and integrated simulation platform for healthcare information system has been discussed aiming to realize automatic (or semi-automatic) mapping of design model to simulation model, with the integration of HDF and specific simulation model specification SMP2, and gradually the simulation approach and integrated support framework for health information systems is established, which can provide flexible decision support and analysis tools for design, integration, optimization, testing of healthcare information system.

\section{REFERENCES}

[1] Research Report on Hospital Informationalization Development in China[R], 2008.5.

[2] Li Baoluo, Li Haiyang. Analysis of Regional Healthcare Informationalization Project Progress in China [J]. China Digital Medicine, 2009.11: 4 9.

[3] Daskalakis, J.Mantas. The Impact of SOA for Achieving Healthcare Interoperability [J]. Methods Inf Med, 2009,(2):190-195.

[4] Ed Seidewitz, Alan Honey. Business Value of Semantic SOA: Standards-based interoperability is not enough [C].SOA in Healthcare: July 12, 2010, Arlington USA.

[5] HL7 Reference Information Model [EL/OB]. Http://www.hl7.org/implement/standards/rim.cfm.

[6] HDF Version 2 package [EL/OB]. Http://www.hl7.org/documentcenter/public/wg/mnm/hdf_workproduct/H DF\%20version\%202\%20package.zip.

[7] Beatriz Perez, Ivan Porres. Authoring and verification of clinical guidelines: A model driven approach [J]. Journal of Biomedical Informatics, 43: 520-536, 2010.

[8] I. Porres, E. Dominguez, B. Pérez, A. Rodriguez, M. Zapata, A model driven approach to automate the implementation of clinical guidelines in decision support systems[C]. in: Proceedings of the 15th Annual IEEE International Conference and Workshop on Engineering of Computer Based Systems (ECBS 2008), pp:210-218,2008. 
[9] A Sciomachen, E Tanfani, A Testi. Simulation models for optimal schedules of operating theatres [J]. International Journal of Simulation, Vol 6, pp26-34, 2005.

[10] RahulC. Basole, Douglas A. Bonder, William B. Rouse. Healthcare management through organizational simulation [OE/BL]. Decision Support Systems, http://dx.doi.org/10.1016/j.dss.2012.10.012, 2012.

[11] TIAN Yan, MA Xiao-pu, ZHANG Xin-gang, ZHANG Ting. Evaluation and Optimization of Clinical Pathway Based on Petri Net [J]. Computer Science, 2013, 40 (5): 193-197.

[12] ZHAO Yan-li. The Hierarchical Timed Colored Petri-Net Based Modeling and Scheduling Optimization of Clinical Pathway [D], Shanghai JiaoTong University, 2010.
[13] Multi-level Evaluation Tool [J]. Journal of Medical Systems, Vol.32, pp: 443-452, Dec 2008.

[14] Elena Tanfani, Angela Testi. A simulation-based decision support tool to analyze clinial pathways in hospital [J]. Advanced Decision Making Methods Applied to Health Care, International Series in Operations Research \&Management Science, Vol 173, 2012, pp: 191-211.

[15] Tanfani Elena, Testi Angela. Improving surgery department performance via Simulation and Optimiaztion[C]. Proceedings of the IEEE Workshop on Health Care Management, Venezia, Italy, pp:1-6, 18-20 Feb, 2010.

[16] Stephen JMellor, Kendall Scott, Axel Uhl, Dirk Weise.MDA Distilled: Principles of Model-Driven Architecture. Addison Wesley, 2004.

[17] European Space Agency, SMP 2.0 Handbook[R], ESOC, 2005. 